\title{
Multidisciplinary educational activity based on optical experiments conducted within an art context
}

Laura Lobato, Josep Vidal, Ariadna Miquel, Angel Lizana, Alba Peinado, et al.

Laura Lobato, Josep Vidal, Ariadna Miquel, Angel Lizana, Alba Peinado, Irene Estévez, Claudio Ramírez, Juan Campos, María J. Yzuel, "Multidisciplinary educational activity based on optical experiments conducted within an art context," Proc. SPIE 9793, Education and Training in Optics and Photonics: ETOP 2015, 97931V (8 October 2015); doi: 10.1117/12.2223182

Event: Education and Training in Optics and Photonics: ETOP 2015, 2015, Bordeaux, France 


\title{
Multidisciplinary educational activity based on optical experiments conducted within an art context
}

\author{
Laura Lobato ${ }^{1, *}$, Josep Vidal $^{1}$, Ariadna Miquel ${ }^{2}$, Angel Lizana ${ }^{1}$, Alba Peinado $^{1}$, Irene Estévez ${ }^{1}$, \\ Claudio Ramírez ${ }^{1}$, Juan Campos ${ }^{1}$, and María J. Yzuel ${ }^{1}$ \\ ${ }^{1}$ Departament de Física, Universitat Autònoma de Barcelona, 08193, Bellaterra, SPAIN \\ ${ }^{2}$ Departament d'educació, Museu d'Art Contemporani de Barcelona, 08001, Barcelona ,SPAIN \\ "laura.lobato.87@gmail.com
}

\begin{abstract}
On the $20^{\text {th }}$ of December 2013, The United Nations (UN) General Assembly $68^{\text {th }}$ Session proclaimed 2015 as the International Year of Light and Light-based Technologies (IYL 2015). The proclamation of an International Year focusing on the light science and applications recognizes the importance of light in the society, which plays a vital role in our daily lives, being visible in a widespread number of different areas, as for instance, in technology, education, energy, art, agriculture, health, among many others.

In this work, the members of the Image Processing Laboratory from the Universitat Autònoma de Barcelona (UAB), analyze the concept of readapting some experiments in optics -usually conducted in different courses at the UAB physics degree- into the artistic context of the MACBA (Museu d'Art Contemporani de Barcelona). This project, called SummerLight, takes place within the framework of the IYL, as part of the activities devised to promote the visibility of light. The readapted experiments are expected to teach and improve the knowledge of high school students with respect to different important physical phenomena related to the wave nature of light as polarization, interferences and diffraction. This study analyzes the suitability of the proposed experiments in terms of student optical skills improvement. In addition, its contextualization into an artistic scenario is also discussed.
\end{abstract}

Keywords: Barcamp, Contemporary Art, innovation, education in Optics, education in Art, Optics experiments, transmission of knowledge, horizontal hierarchy.

\section{INTRODUCTION: A NEW LEARNING FORMAT}

All children have the capacity to innovate, think and express divergent thoughts. These capacities are sometimes lost when children grow up, probably due to the education systems which do not promote or reward these skills but rather, tend to stifle them. Students are assessed -and ultimately selected- depending on some criteria which are identical for all the children and imposed by those who design the education plans. That is, students usually lose their individuality and since the learning process is often away from the children's own reality and experiences, they eventually lose their interest on knowledge. In the case of Physics and Optics, the loss of creativity and interest is of vital importance. How do we want to develop scientists if we are killing the innate creative scientists that most children are?

According to H. Gardner, a well-known scientist from Harvard University, the creativity is an endeavour which "requires deep immersion in the subject matter, the development of skills, the capacity to ask questions that haven't been asked before and to spend as much time as necessary to come up with the best answers we can to those questions" [1]. He also points out the fact that if one wants to be creative, one must take risks and do not care if they fail. For this to happen

Education and Training in Optics and Photonics: ETOP 2015, edited by Eric Cormier, Laurent Sarger Proc. of SPIE Vol. 9793, 97931V · C 2015 SPIE, IEEE, OSA, ICO · doi: 10.1117/12.2223182 
there must be a place where experimentation is tolerated and even fostered. The attitude of educators is also of importance. When they pose questions to which there are many answer and when they give students the chance to make errors, they are promoting a learning based on creativity. J. M. Davis [2] agrees with this idea and points out the fact that creativity flourishes in environments where autonomy, openness, and collaborative relationships are valued.

M. Grossen [3] talks about the concept of "creative collaboration" which, according to him, refers to two different aspects: the fact that collaboration in itself is a creative task, and the fact that the outcomes from study groups usually tend to be more creative due to the exchange of ideas and the constant feedback among the team members. This concept is of particular importance in science, where most of the studies are the result of teamwork.

Coming back to education, many pedagogues $[4,5]$ agree with the idea that society and science have radically changed in the last century and still continue to change at a high rate in the $21^{\text {st }}$ century and in this sense, new education methods are required. Education should prepare next generations for the future challenges and foster the human progress. However, the issue remains difficult. How is the knowledge transmitted? Which are the factors which will allow building more creative and therefore more efficient education systems? In this article, we present an innovative Optics-and-Art based education programme format, intended to high-school students from different study areas. The programme has been developed at the MACBA and counts on the support of the UAB.

\section{THE PRECEPTS OF SUMMERLIGHT}

SummerLight is an educative project in which Optics experiments from the UAB will be presented within an art context (at the MACBA installations). The proposed plan tries to break away from pre-established education programmes and commits to the horizontal transmission of knowledge (in this case both Optics and Contemporary Art knowledge) rather than to the knowledge coming from the authority and with a marked vertical hierarchy. The idea is to implement a more transversal method where students have an active role in their own learning process. In this programme, the Optical experiments are of great importance since they allow students to understand complex concepts from an interactive and hand-on approach.

Summerlight has been inspired by the BarCamps ${ }^{1}$, giving special consideration to the idea of the "student maker" who learns on a trial/error basis. The interaction among students is also very important since the students' different knowledge, skills and interests lead to the enrichment of the group as a whole and the students as individuals. SummerLight aims at the collective building of Optics and Art knowledge and culture. The understanding of the optical concepts comes therefore, not from repetition, but from the creation and the association of ideas. The main pedagogical traits are:

- Horizontal hierarchy, or the absence of hierarchy

- Hands-on approach

- Team work

- Work flexibility, i.e., students make their own choice about the working subjects. Teams change depending on the activity

- Active participation of students in several simultaneous activities, some of them directly focus on the pedagogical objective and some of them related to the general performance of the course

- Collaborative learning system

\footnotetext{
${ }^{1}$ BarCamp is a course format where participants propose and develop, in groups, their own ideas. These courses were first performed in the field of computer programming, but because of their versatility and the advantages they offer, these were diversified to other domains, such as art, for instance.
} 
- Make the most of all the opportunities: low-cost material, the use of installations, etc.

More in particular, SummerLight has been conceived within the framework of the International Year of Light and specially shaped for thirty high-school students -17 and 18 years old-. The project consists on a one-week duration program during which the students are expected to explore and learn about Optics. This initiative has been specially devised assuming the fact that Optics concepts, although increasingly present in our daily lives, are usually difficult to explain to students and also difficult to understand by them. In this sense, we have decided to design a different and innovative format course where Optics is explored from an artistic approach. Because Optics is a very tangible and visual science, the possibilities that art generates are infinite. We next present the learning model of SummerLight, which has been based on the Kanter's model of innovation [6]:

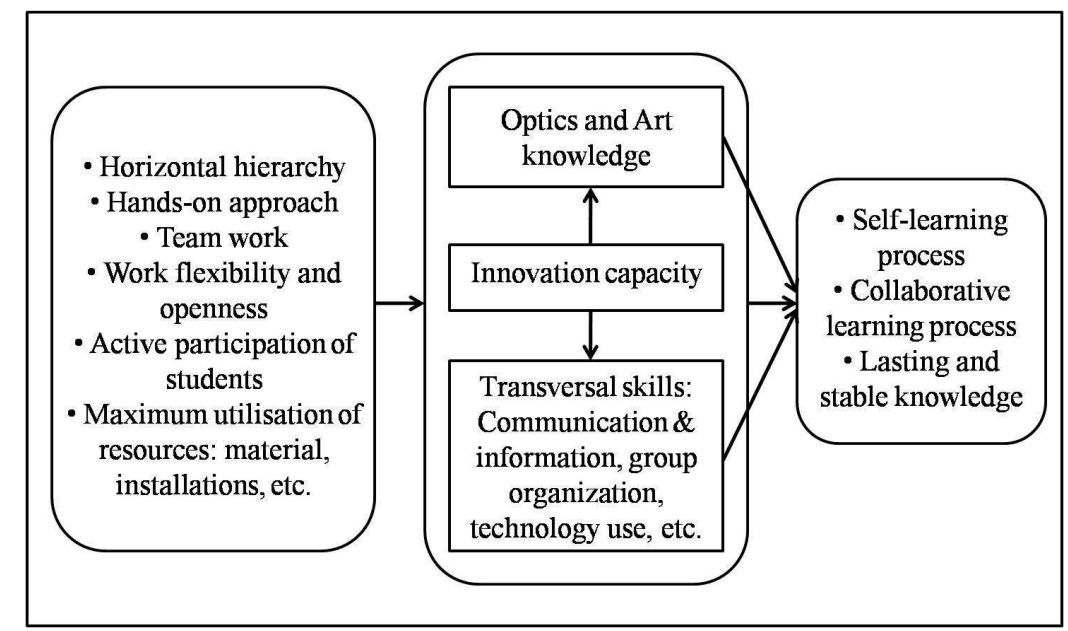

Figure 1. Learning model of SummerLight, in which different resources are mainstreamed to obtain an innovative capacity. This leads to the specific knowledge in Optics and Art, but also to other transversal skills. These capacities are again streamed, obtaining a lasting knowledge which is more efficient.

\section{STRUCTURE OF SUMMERLIGHT}

The course is articulated at two different levels, regarding the form and the working methodology. These two organizational levels are simultaneously exhibited and they influence each other, giving place to a complex structure.

With respect to the form, SummerLight has been conceived as a "Consultancy firm specialized in light". Every day, students receive questions related to light and Optics'. These questions must be all of them answered within 24 hours. The process is the following: when they listen to the questions, students themselves decide in which questions they prefer to work, immediately establishing the working groups which will operate this particular day. These groups will most surely change the next day, since students will find new interests in the cluster of questions newly arrived. The working groups decide then how they are going to answer the chosen questions. They propose some experiments and have a limited time to perform them. Once the answers are found, they communicate to the person who previously called to tell him the response.

We highlight the importance of the knowledge transmission in this particular format. The only communication channel is auditory, both in the listening of the questions and the transmission of the answers. The denial of any visual support is an

\footnotetext{
${ }^{2}$ These questions are real questions posed by citizens from Barcelona. People will phone and leave their questions in an answering machine. These questions will be answered within 24 hours by the specialists, who are the SummerLight students.
} 
important restriction which may a priori make difficult the communication, but which fosters the innovation process and makes students to find new ways to express themselves. At the same time, there is also a kind of "Wikilight" which is made up from the students' answers ${ }^{4}$.

The programme presents therefore three different moments: listening to the questions and formation of the working groups as well as planning of the work to be performed, experimental development in which students carry out their own proposals and finally, delivery of the answers by phone. The idea is that all the students can finish the course having comprehended a number of concepts in Optics and Contemporary Art. To ensure that this happens, another activity has been included in the programme, which is called "Fundamentals". Students listen to specific concepts through headphones and must then explore them. They can perform experiments, think about a device related to the particular concept, etc. They are supposed to post an entry into the "Wikilight", which implies an interesting transference from heard knowledge transmission (headphones) to audiovisual knowledge transmission (Wikilight).

Regarding the methodology, the activities are completely determined by the working conditions, which have been intentionally brought to the extreme. Again, a restriction has been imposed which fosters the creativity of students and their capability to problem-solving. This restriction is the amount of light available. Through the course, the degree of light significantly changes. Students begin the course in complete darkness, and as the course advances, more light is allowed. Optics experiments will be completely conditioned by this parameter.

\section{SUMMERLIGHT: A PLACE FOR INNOVATION, A PLACE FOR OPTICAL EXPERIMENTS}

We have already pointed out the importance of the working place to achieve innovation, creativity and a collaborative learning process. The room must be a unique ambience, which will allow for the following aspects:

- Interaction among the different working groups.

- All the groups have to share the same material. This is another added challenge, since they will have to look for different approaches when they cannot use the material they wanted at first instance.

- We ensure that all the students work with the same light conditions.

As already pointed out, some optical experiments from the UAB will be brought to the SummerLight programme. Students will be able to use the setups either to answer the questions or to explore about the concepts previously heard through the headphones. The experiments have been chosen specifically due to the tangibility with which they expose Optical concepts which otherwise may be difficult to understand. In particular, experiments of polarization, diffraction and interferences have been included. In addition, the outcomes from these experiments present very visual effects. We next present each of these experiments:

\subsection{Fraunhofer diffraction:}

In this experiment, light is understood as a wave. Diffraction is a phenomenon produced when light crosses a small aperture (in the order of its wavelength) since in this situation, the light wave is perturbed. The Fraunhofer diffraction is a particular case of the Fresnel diffraction that occurs when light source illuminating the aperture and the observational plane are both of them faraway from the aperture (also called object), so light can be approximated to plane waves.

\footnotetext{
${ }^{3}$ With this term we refer to a kind of blog where students can all of them include their own comments. The idea is to build something similar to Wikipedia, where the knowledge is collaboratively created.

${ }^{4}$ However, the communication with the "client" who asks the question is never made through the "Wikilight", but rather through the phone. The visual support will serve to another purpose of the programme which we will explain later on in this paper.
} 
Under this approximation the diffraction pattern produced by the diffractive object in the far-field can be understood as the modulus squared of the Fourier transform of the diffractive object [7].

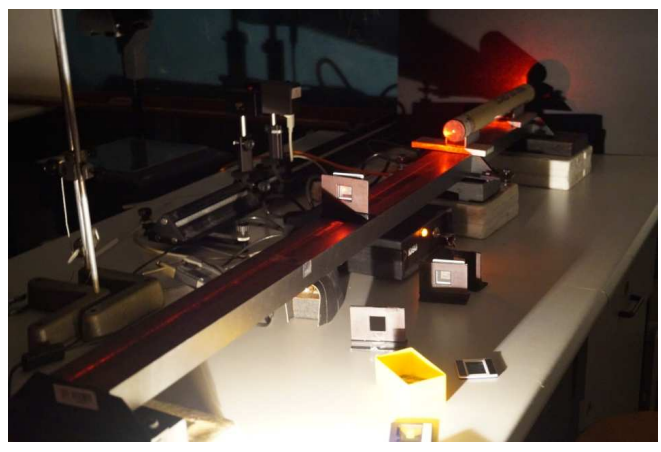

Figure 2. Set-up of the Fraunhofer optical experiment. The laser ray (He-Ne) crosses a small object, whose diffraction is projected on a faraway screen. The diffraction pattern corresponds to the Fourier transform.

We count on different objects, whose diffraction patterns can be seen in the following table. Note that for the single slit, the double slit and the circle, the mathematical descriptions have been included, where $\vartheta$ refers to the angle which determines the position that we are analyzing on the far-field, where a screen has been placed. For the other two objects (the hexagonal grid of hexagons and the squared grid of squares, respectively), we have not included the mathematical description because of its complexity.

Table 1. Object, Fraunhofer diffraction pattern observed at the screen and its corresponding mathematical description.

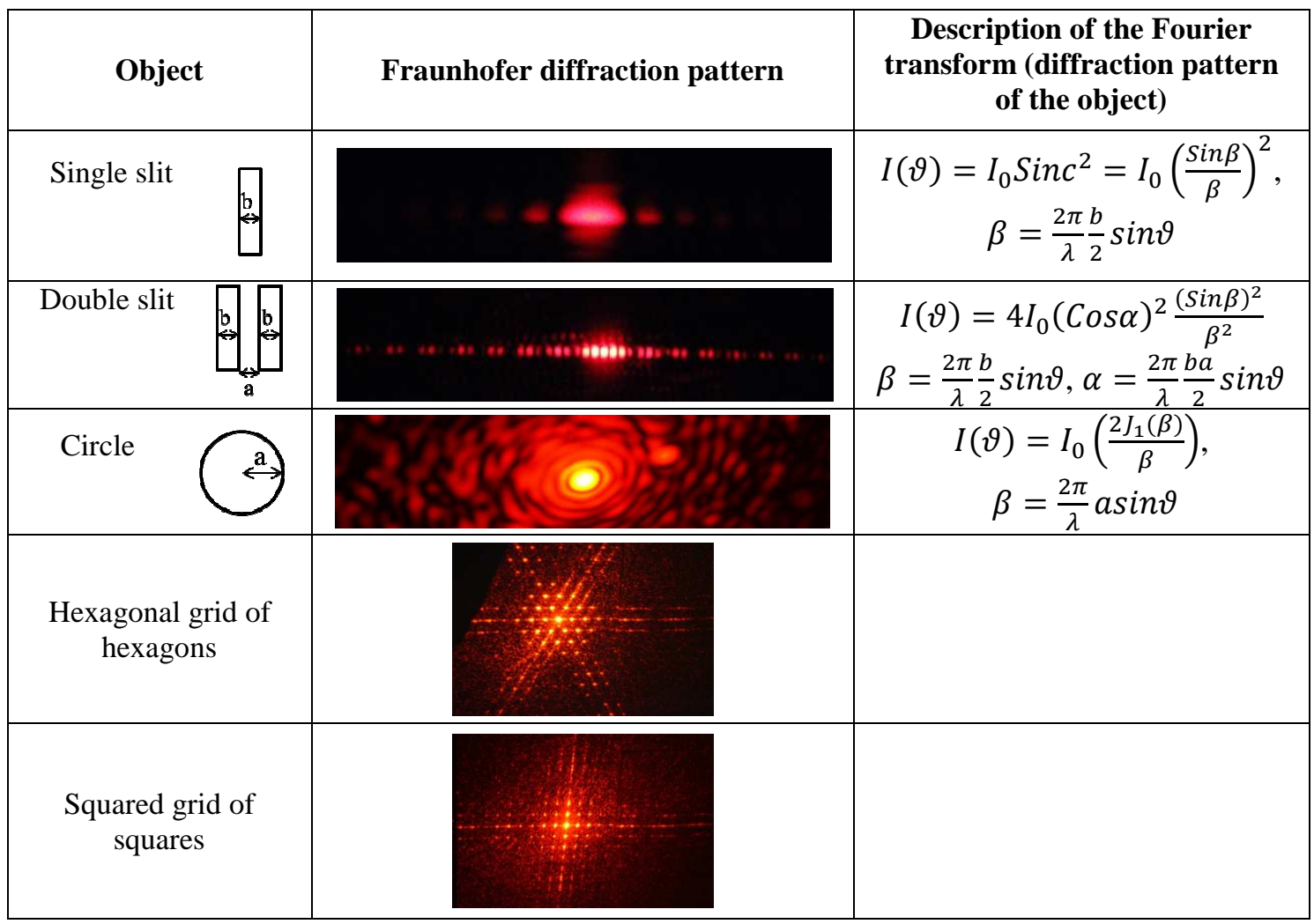


The main idea of this experiment is that students understand the wave-like behavior of light and the consequent diffraction phenomena produced by small apertures due to spatial discontinuities (in intensity or phase) that they impose. A speech should reinforce the importance of this physical phenomenon, explaining not only the fundamentals of diffraction but also that it can be used as a tool in many applications. Clearly, all the applications in the field of diffractive optics are based on diffraction [8], as for instance for the generation of digital lens [9] and for the generation of filters to improve the quality of images [10]. Nevertheless, diffraction is useful in many other fields as for instance in biology [11] (e.g., to track and measure bacteria from the diffraction pattern they produce) and for the characterization of the structure of crystalline materials by using X-ray diffraction.

\subsection{Polarization}

When the wave behavior of light was experimentally proved (Young experiment), several scientists started to study light as wave, unrevealing its polarization properties. Therefore, different mathematical formalisms were proposed [12, 13] to describe the polarization of light as well as its interaction with polarizing mediums and surfaces. Light can be understood as an electromagnetic wave formed by an electric field and a magnetic one, which are always perpendicular to each other and also to the propagation direction of light. Most of light sources (such as the Sun, a light bulb, etc.) emit unpolarized light (i.e., the relative phases and amplitudes of the electromagnetic components cannot be defined as they randomly vary). Other light sources as for instance most of lasers, are fully polarized. In addition, a fully unpolarized beam can become partially or fully polarized when interacting with different materials. In case of fully polarized light, depending on the relative phases and amplitudes of the constituent components of the electric field, we can distinguish three different types of polarization states: linear polarization (the electric components of field oscillates in phase or contraphase), circular polarization (the electric components of the light oscillates with the same maximum amplitude but they are retarded $\pm \pi / 2$ ) and elliptical polarization (any other case). More specifically, linear and circular polarizations are particular cases of elliptical polarizations [13]. In addition, if the state of polarization of light beam is not constant but varies as a function of space and/or time, some un-polarized content may be present, defining partial polarized beam, or even fully un-polarized beams.

As stated, a fully unpolarized light beam can become partially or fully polarized when interacting with some materials that can be found in different states of the matter. For instance, natural light is polarized by crossing dichroic materials (e.g., Polaroid films), by reflection on dielectrics when impinging the surface under the Brewster's angle, or due to the Rayleigh's diffusion in some gases. On the other hand, the state of polarization of the light beam can be transformed into a different one by being transmitted through birefringent materials. These materials present different dielectric responses (they present structural anisotropy) which lead to a very interesting optical property: they show different refraction indexes (two different index for uni-axial materials and three different indexes for bi-axial materials). In this section, we focus both on the study of polarization by dichroism and on the retardance introduced between the components of an electric field when passing thought birefringent materials.

Polarizers are dichroic materials that can polarize light beams. In these thin films, molecules are aligned, forming chains of molecules which function as a dipole and will therefore absorb light in the direction of the chain. One component of the light will be almost completely absorbed while the perpendicular component will pass through the polarizer. The efficiency of the polarizer (i.e, its absorption and transmission capacities) always depends on the material, its thickness and the wavelength used. In the ideal case, by crossing the transmission axis of two polarizers, the screen should be dark, but due to the efficiency of the polarizers, which is never perfect, we can observe a bluish color on the screen. To analyze the efficiency of a dichroic material as a function of the wavelength, different filters can be placed after the white light, leading to more monochromatic light source. 


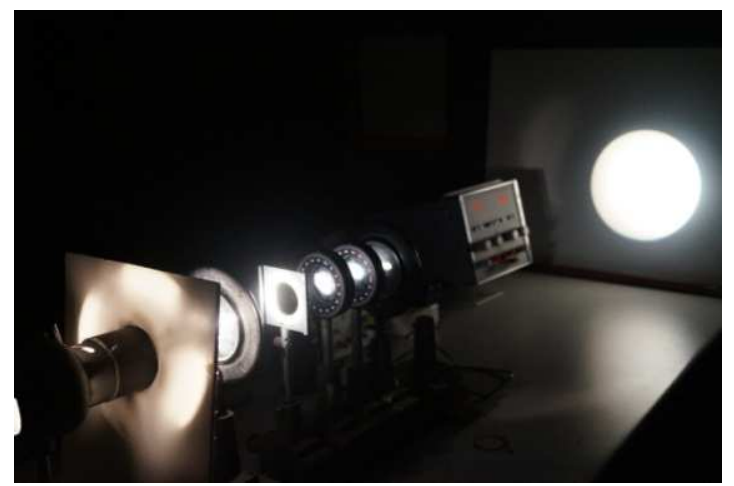

Figure 3. Polarization set-up. We illuminate with an extended white light source. We next place a convergent lens at a proper distance, leading to an approximately collimated beam. A second convergent lens is placed at the exit to image the sample plane. Between them, we can place the different materials to be studied (polarizers, birefringent materials, etc.).

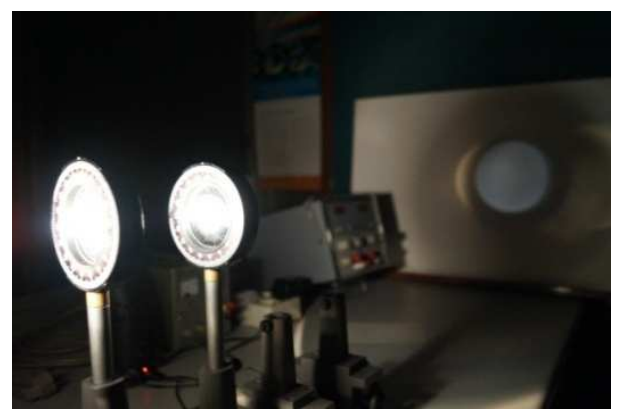

Figure 4. Two crossed polarizers result in a bluish screen since the efficiency of polarizers is not one hundred per cent and depends on the wavelength of the light. These polarizers are less efficient for the blue part of the spectrum.

We also study polarization in birefringent materials. Birefringent materials are optically anisotropic since light travels with different speed depending on the axis. The phase of one component will be retarded with respect to the other one. The shift of phase between the two perpendicular polarization components, $\delta$, depends on the difference of the refractive indexes between the ordinary and the extraordinary axis, $\left(\mathrm{n}_{\mathrm{e}}-\mathrm{n}_{0}\right)$, the width of the material, $\mathrm{d}$, and the wavelength of the light with which we illuminate, $\lambda_{0}$. Mathematically it can be expressed as:

$$
\frac{2 \pi}{\lambda_{0}}\left(\mathrm{n}_{\mathrm{e}}-\mathrm{n}_{0}\right) \mathrm{d}=\delta
$$

To study the effect of birefringent materials on the polarization of light, we use the set-up provided in Fig. 4, by setting the transmission axis of the two polarizers parallel one to each other. After this, a birefringent material is placed in between the two polarizers and a convergent lens images the sample plane to a screen. As an example of birefringent material, we have used a set of cellophane strips stuck on a glass wafer and with the extraordinary axis oriented at the same direction. Images in Fig. 5 show the light distribution obtained at the screen when illuminating the cellophane with linear polarization having different orientations. In Fig. 5A) we use a linear polarization with the same orientation than the extraordinary axis of the cellophane strips. In this situation, the state of polarization is not modified because the entire electric field is travelling into an effective media of index $n_{e}$. Thus, a uniform white light image of the cellophane is observed at the screen. We next illuminate the sample with linear polarization but in this case out of the extraordinary axis, this being achieved by rotating the orientation of the input polarizer. In this situation, a retardance $\delta$ is introduced between the electric field components, the value of $\delta$ being given by Eq. (1). As a consequence, the color patterns shown in Figs. 5 B) and C) are observed. To understand these color distributions, note that we are illuminating the cellophane 
strips with polychromatic light, and thus each wavelength sees a different retardance. In this case, each wavelength presents a different polarization at the exit of the sample plane. Afterwards, this set of polarizations (a polarization for each $\lambda$ ) is projected on a privileged polarization, this polarization being fixed by the polarizer placed at the exit (the linear analyzer). In addition, note that on the strips edges (top and bottom) we observe a change of color. This is because the sample consists on different superposed cellophane layers, leading to different thicknesses $d$ at the edges (i.e., to different values of $\delta$ in eq. (1)).

We can also observe the birefringent response in plastics when they are subjected to an external stress. In fact, stress in plastic may induce structural anisotropy, thus turning the material into a birefringent material (see Figure 6).
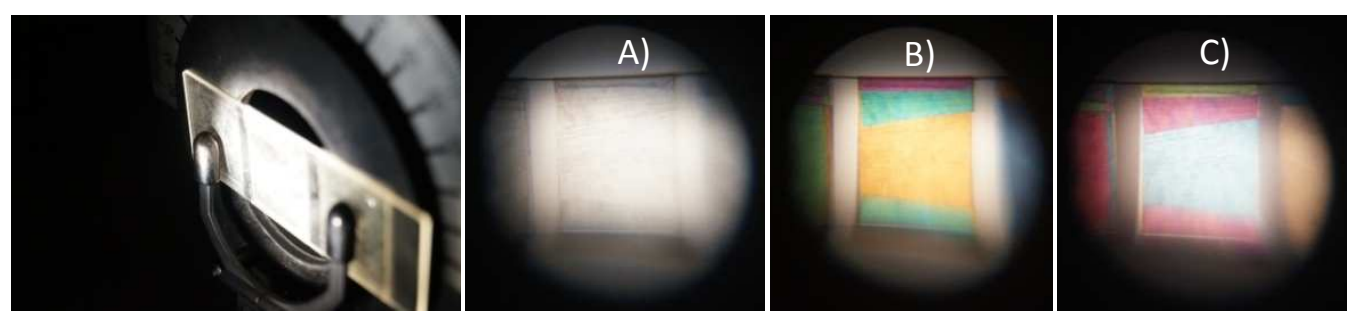

Figure 5. Left image: Illumination of cellophane with white light. The birefringent material has been placed between two polarizers. Depending on the position of the second polarizer with respect to the first one, different wavelengths are selected, giving place to the different polarization patterns that we can observe in the three consecutive pictures. A) For the transparent pattern, light crosses the material in such a way that the incident linear light is parallel to one of the anisotropic axis of the cellophane. In this case, there is no shift of phase between the two components and the polarization of light is not modified. B) and C) Different colors are obtained with the superposition of layers of cellophane, changing the parameter $\mathrm{d}$ in equation (1).

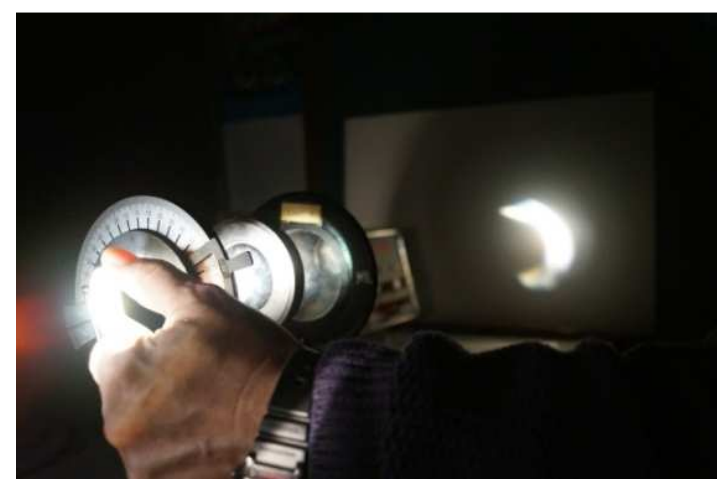

Figure 6. We place a plastic material between the two crossed polarizers and exert a pressure on the material, changing the chain of molecules. This turns the plastic into a birefringent material.

\subsection{Michelson interferences}

Interferences are another physical phenomenon related to the wave-like behavior of light. In the following experiment, the Michelson interferometer is studied. This experiment was initially performed by Michelson because he wanted to demonstrate the existence of the aether (theoretical medium that was used by physicists to explain the propagation of light, in a model similar to what sound waves do in the air). According to Michelson, in presence of aether, the interference pattern produced by the superposition of two light rays should be different depending on the orientation of the interferometer with respect to the Earth translation. After many set-up improvements, it was finally concluded that aether does not exist since interferences were the same independently of the chosen orientations for the Michelson 
interferometer. However, the interferometer has been since then widely used for metrology because of its high precision. For example, we can know the width of a thin film by knowing the refraction index of the material and vice versa.

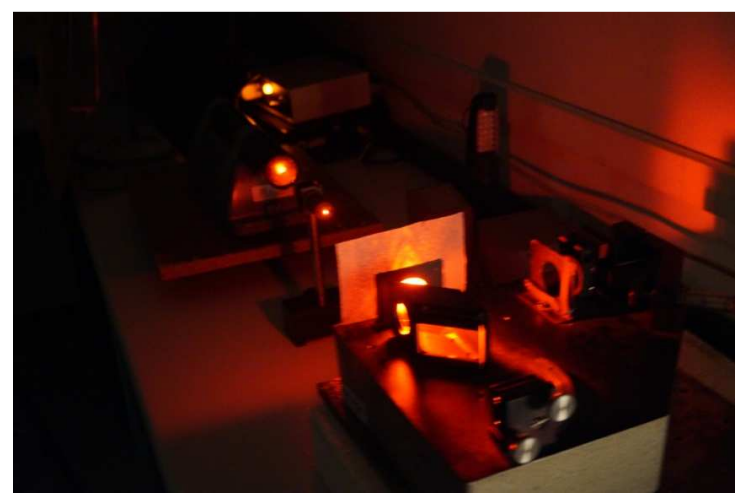

Figure 7 Set-up of the Michelson interferometer. We can observe two arms whose mirrors can be moved in order to change the distance that light travels in each arm, creating therefore the interference pattern. For illuminating the interferometer, we use a HeNe laser followed by a microscopic objective which creates a punctual light source. The obtained interferences exhibit rotation symmetry.

By using monochromatic light we are ensuring large time coherence for the light. In addition, since we are using a punctual light source, the obtained interference pattern is delocalized. In this configuration, spherical waves are produced. If the system introduces a difference in the optical paths of the interfering beams, the interference pattern shows rotational symmetry. A series of maximums and minimums of intensity are distributed in concentric rings. The larger the optical path difference, the thinner and closer the rings will appear. If the case where the optical path is identical for the two arms, a constant intensity will appear. However, if a misalignment exists between the two mirrors of the Michaelson interferometer, a different fringe pattern will be observed.
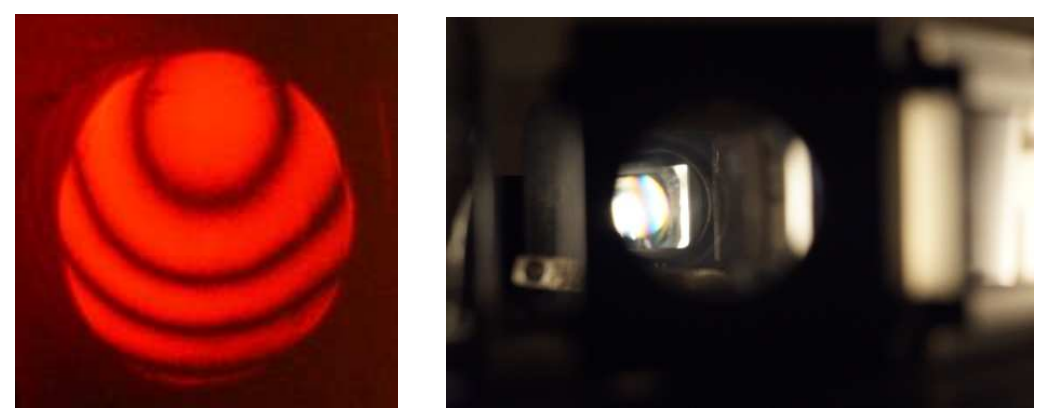

Figure 8. Left: Ring-like interference pattern obtained when illuminating the interferometer with a punctual and monochromatic $(633 \mathrm{~nm})$ light source. Right: Fringes-like interference pattern obtained by illuminating the interferometer with an extended whitelight source.

Finally, the concept of temporal coherence can be studied by using a polychromatic light source. To this aim, we have illuminated the system with an extended incandescent lamp. As white light presents a very short temporal coherence, interferences can only be observed when the two arms optical paths are very similar to each other. In addition, similarly to what happens in the previous case when dealing with extended light, the interference pattern is also localized. Under this scenario, when interferences are observed by using white light, a very good calibration of the Michelson interferometer is ensured. In this particular situation, the interferometer can be used to measure the thickness or the refraction index of different thin films by measuring the optical path difference introduced by the material. 


\section{CONCLUSION}

We have presented an innovative education programme based on Optics and Contemporary Art which tries to promote the long-lasting knowledge, the self-learning process of the person as well as the collaborative learning. Some chosen Optics experiments from the UAB have been assembled at the installations of the MACBA (where the project is performed) because of their particular characteristics. These experiences are easy to perform by 18-years old high-school students and present remarkable visual outputs which make them very impressive. With the appropriate guidance from the monitors, we can presume that students will learn about diffraction, polarization of light and interferences which are all of them physical phenomena related to the wave nature of light. The relationship between Optics and Contemporary Art has an important role in the progress of the programme.

The form and methodology proposed in SummerLight allow students to get involved in subjects they are interested in, fostering the active participation of them in their own learning process. The programme counts on several innovative aspects. For instance, regarding the form, the course has been devised like a consultancy firm with expertise in light, where supposedly, students are the light experts and must therefore answer real questions about any wave-like phenomenon of light. The answers must be given within 24 hours from the moment someone asked them, and to do this, they have to perform any experiment they can envisage. Regarding the methodology, the degree of light in the working room changes as the course advances, starting at absolute darkness. Special importance has been given to the transmission of knowledge, which is mainly done through auditory meanings (although visual knowledge transmission is also present through the "Wikilight").

The final goal of SummerLight is to build a learning process which is more effective and where students can acquire problem-solving capacity. We strongly believe that the multidisciplinary approach in which science is addressed and the openness with which ideas are considered during the whole programme will encourage students to pursue hard science studies, and particularly, Physics and Optics studies. We hope that this innovative course can be an inspiration for other educative programmes and that we can increasingly break away from pre-established education models in which students do not take an active role in their own learning.

\section{REFERENCES:}

[1] H. Gardner. "Getting at the Heart of the Creative Experience", LEARNing Landscapes, Vol 6, No. 1 (2012).

[2] J.M. Davis. "Supporting creativity, inclusion and collaborative multi-professional learning", Improving Schools, vol 16, 1, p. 5-20 (2013).

[3] M. Grossen. "Methods for studying collaborative creativity: An original and adventurous blend", Thinking Skills and Creativity, 3, p. 246-249 (2008).

[4] V-A. Enăchescu and C. Damasaru. "Innovation and Creativity in Human Resource Ma-nagement. Why should we change the way we teach our students?", Review of International Comparative Management, Vol 14, 4 (2013).

[5] M.A.D. Likeschová and A. Tichá. "Multicultural education, creativity and innovation at universities in the Czech Republic", Social and Behavioral Sciences, vol 93, p. 349-355 (2013).

[6] M. Mehta, A. Chandani and B. Neeraja, "Creativity and Innovation: Assurance for Growth", Procedia Economics and Finance, vol 11, p. 804-811 (2014).

[7] Campos, J. and Yzuel, M.J., 'Procesado óptico de la información', [Óptica Avanzada], Ariel Ciencia, Barcelona, 155-206 (2002).

[8] A. Lizana, A. Márquez, L. Lobato, Y. Rodange, I. Moreno, C. Iemmi and J. Campos. "The minimum Euclidean distance principle applied to improve the modulation diffraction efficiency in digitally controlled spatial light modulators", Optics Express, vol 18, 10 (2010). 
[9] L. Lobato, A. Márquez, A. Lizana, I. Moreno, C. Iemmi and J. Campos. "Characterization of a Parallel Aligned Liquid Crystal on Silicon and its application on a Shack-Hartmann sensor", SPIE Proceeding, vol 8 (2010). [10] A. Márquez, C. Iemmi, J. Campos, J.C. Escalera, and M.J. Yzuel, "Programmable apodizer to compensate chromatic aberration effects using crystal displays. Optimization of the modulation diffraction efficency", Opt. Exp. 13(6), pp. 2111-2119, (2005).

[11] A. Arcovito, T. Moschetti, P. D'angelo, G. Mancini, B. Vallone, M. Brunori and S. Della Longa. “An X-ray diffraction and X-ray absorption spectroscopy joint study of neuroglobin", Archives of Biochemistry and Biophysics, vol 471, 1, p. 7-13 (2008).

[12] R. C. Jones, "A new calculus for the treatment of optical systems," J. Opt. Soc. Am. A 31(7), 488-493 (1941).

[13] D. Goldstein, Polarized Light, (Marcel Dekker, NY, 2003). 